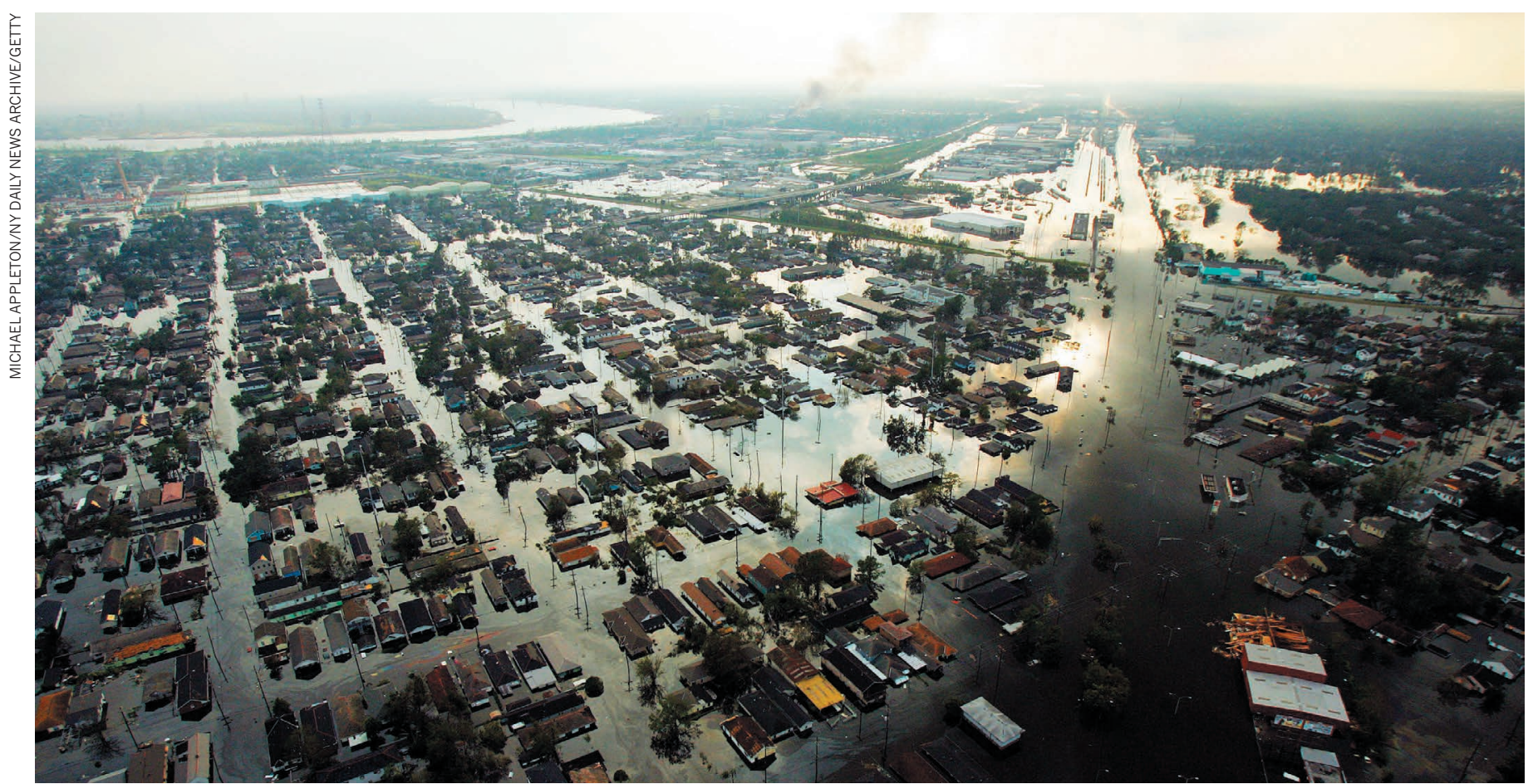

Figure 1 | Flooding in New Orleans, Louisiana, after Hurricane Katrina, 2005. Brown et $_{\text {al. }}{ }^{2}$ report that flooding events, such as that caused by Hurricane Katrina, will affect more land and people in the future as a result of sea-level rises associated with global warming.

not consider the effects of coastal-protection measures, such as the construction of dykes or dunes. If such measures were considered in the analysis, then the area and number of people exposed would change. Moreover, the construction of coastal defences will be largely driven by economic considerations, which will be different for different countries.

The authors define land at risk from sealevel rise as the 1-in-100-year coastal floodplain. But will people be driven away from such land by the infrequent floods, or will they accept the occasional inundation, moving away only temporarily as needed? It might be better to consider the area of land that will become permanently flooded to make a moredirect estimate of the population that will be exposed to sea-level rise.

A limitation of the study is that Greenland, Antarctica and all glaciers elsewhere are lumped together as the land ice that contributes to sea-level rise. However, ice will probably melt at different rates in each of these regions. This will cause the relative contributions of the different sources to change over time. One way to improve the regional estimates of sea-level rise would therefore be to scale the contributions of the ice sheets according to their individual effects.

Nevertheless, studies such as those of Brown and colleagues are essential, because they show the complexity of the climate system's response to change and how this affects society. By directly connecting the effects of climate change to the consequences for humans, the authors clearly show that climate mitigation needs to happen now for a better future.
Aimée Slangen is in the Department of Estuarine and Delta Systems, NIOZ Royal Netherlands Institute for Sea Research and Utrecht University, 4401 NT Yerseke, the Netherlands.

e-mail:aimee.slangen@nioz.nl
1. McGranahan, G., Balk, D. \& Anderson, B. Environ. Urban. 19, 17-37 (2007).

2. Brown, S. et al. Earth's Future 6, 583-600 (2018).

3. Goodwin, P. Clim. Dyn. 47, 2219-2233 (2016).

4. Slangen, A. B. A. et al. Clim. Change 124, 317-332 (2014).

5. Vafeidis, A. T. et al. J. Coast. Res. 24, 917-924 (2008).

\title{
GENE REGULATION
}

\section{A new phase in transcription}

\section{A subunit of the enzymatic complex $\mathrm{P}$-TEFb can induce compartmentalization of proteins into liquid-like droplets in cells. This phase separation might help P-TEFb to promote gene transcription. SEE LETTER P.318}

\section{JAMES A. GOODRICH \& DYLAN J. TAATJES}

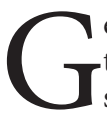
enomic DNA is not indiscriminately transcribed into RNA. Instead, select sequences are transcribed at any given time or in any given cell type. How this process is regulated has been an active area of research for decades, because of its complexity and importance in embryonic development and disease. On page 318, Lu et al. ${ }^{1}$ describe an aspect of transcription regulation that involves liquid-liquid phase separation - a process by which proteins, nucleic acids and other molecules self-organize into liquid-like droplets to enable subcellular compartmentalization.

Transcription is catalysed by the enzyme
RNA polymerase II (Pol II). The activity of Pol II is, in turn, regulated by a complex called $\mathrm{P}-\mathrm{TEFb}$, which consists of the protein cyclin T1 (CCNT1) and the kinase enzyme CDK9. P-TEFb binds to the carboxy-terminal domain (CTD) of Pol II (ref. 2) - this domain consists of 52 repeats of 7 amino acids, 5 of which can be phosphorylated. The CCNT1 subunit regulates the activity of CDK9, which can phosphorylate the Pol II CTD many times to help control Pol II function ${ }^{3}$.

Lu et al. first investigated which region of CCNT1 is responsible for regulating CDK9 activity. They found that mutations in a domain of CCNT1 rich in the amino acid histidine led to defects in the activation of 


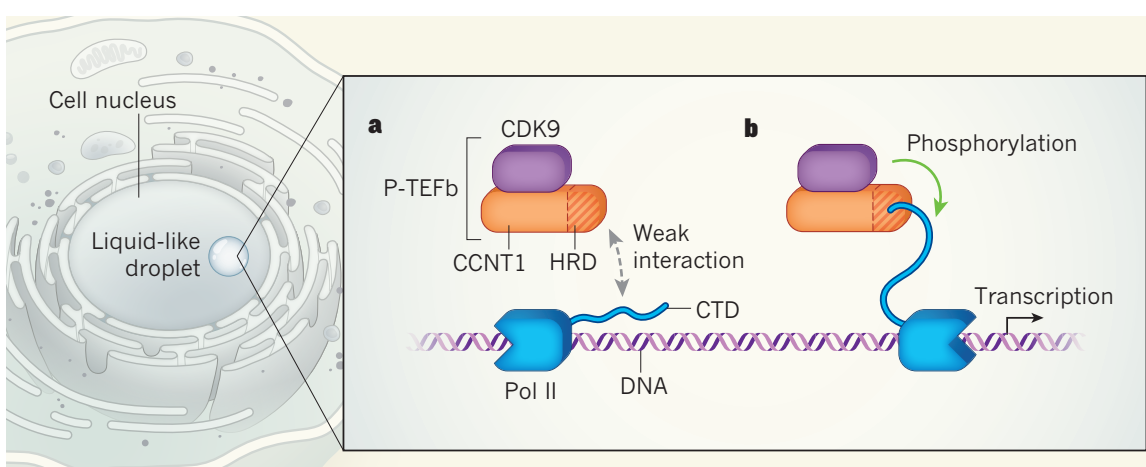

Figure 1 | Phase separation in transcription regulation. a, The P-TEFb complex consists of two proteins, CCNT1 and CDK9. Lu et al. ${ }^{1}$ have identified a histidine-rich domain (HRD) in CCNT1 that promotes liquid-liquid phase separation - a process in which P-TEFb and the enzyme RNA polymerase II (Pol II) coalesce inside a liquid-like droplet in the cell nucleus, within which they weakly interact. b, The authors provide evidence that these weak interactions within phase-separated droplets enhance direct, functional interactions between P-TEFb and Pol II that allow CDK9 to phosphorylate the carboxy-terminal domain (CTD) of Pol II, promoting the ability of Pol II to transcribe DNA.

Pol II-mediated transcription in vitro. Notably, removal of this histidine-rich domain (HRD) decreased phosphorylation of the full-length Pol II CTD by P-TEFb, but had no effect on phosphorylation of a truncated, nine-repeat version of the CTD. The Pol II CTD is known to undergo phase separation into liquidlike droplets ${ }^{4}$, but this ability declines as the length of the CTD decreases ${ }^{5}$. The authors' results therefore hinted that the HRD might be involved in phase separation, and that this process might improve the ability of $\mathrm{P}-\mathrm{TEFb}$ to phosphorylate the full-length Pol II CTD.

The researchers then fused CCNT1 to a DNA-binding domain that directed it to sites at which transcription begins. This fusion strategy allowed the group to evaluate the function of CCNT1 on its own, away from other proteins that would typically guide it to DNA. The fusion protein activated transcription, but activation decreased if HRD was deleted. This finding further supports the idea of CCNT1 being involved in phase separation, because other proteins that induce phase separation behave similarly if fused to DNA-binding domains ${ }^{4}$. Presumably, Pol II is recruited into phase-separated droplets formed by CCNT1, thus concentrating Pol II around DNA and promoting the activation of transcription.

Next, Lu et al. carried out a series of experiments that demonstrated that the HRD of CCNT1 does indeed promote phase separation in vitro and in cells. As expected for a domain that serves a key regulatory function, the CCNT1 HRD is evolutionarily conserved among vertebrates. Moreover, the authors found that a similar domain in a kinase called DYRK1A also regulated that enzyme's activity and promoted phase separation.

Finally, the group investigated whether the HRD influences the location and dynamics of CCNT1 in the nuclei of living cells. Using two complementary techniques to track CCNT1 movement, the researchers provided evidence that the presence of the HRD facilitates the retention of CCNT1 at actively transcribing genes. Consistent with this finding, CCNT1 formed concentrated clusters called speckles in the cell nucleus in an HRD-dependent manner. These speckles were dispersed by 1,6-hexanediol - a hydrophobic compound that disrupts phase separation.

Lu and colleagues' work suggests that the $\mathrm{P}-\mathrm{TEFb}$ complex can trigger the formation of liquid-like droplets around transcriptionally active genomic regions. However, P-TEFb does not require phase separation to interact with the Pol II CTD (ref. 2), and so it will be challenging to determine the exact contribution of phase separation to P-TEFb's function in transcriptional regulation. The authors show that the HRD augments the ability of CCNT1 to directly interact with the Pol II CTD. This observation can at least partially explain the defects in transcription caused by HRD mutations, but changes in phase separation are probably also involved.

The authors propose that compartmentalization through phase separation, which involves multiple weak interactions between proteins within a droplet, might concentrate $\mathrm{P}-\mathrm{TEFb}$ and Pol II together. This, in turn, probably promotes functional interactions between $\mathrm{P}-\mathrm{TEFb}$ and Pol II, leading to highly efficient phosphorylation of the CTD (Fig. 1). In this way, phase separation could ensure robust regulation of gene-expression programs, and enforce highlevel activation of genes to enable rapid or sustained responses to extracellular stimuli.

Lu and co-workers' study raises many interesting questions. For example, do $\mathrm{P}-\mathrm{TEFb}$ complexes selectively associate only with nuclear phase-separated domains that contain Pol II? Or can P-TEFb also associate with phase-separated droplets that are devoid of Pol II? A transcriptional repressor protein called HP1a can also promote phase separation $^{6,7}$. Thus, phase-separated domains seem to demarcate both transcriptionally active and transcriptionally inactive genomic regions. It remains to be seen whether the HP1 $\alpha$ and the P-TEFb compartments are biophysically distinct, and whether each can selectively exclude specific sets of proteins, nucleic acids or metabolite molecules to effectively repress or promote transcription, respectively.

The impact of CCNT1 HRD mutations on global gene-expression patterns also remains to be defined. On the basis of current mod$\mathrm{els}^{8}$, it seems plausible that highly expressed genes will be most sensitive to HRD mutations, because their elevated expression might require high local concentrations of Pol II and transcription factors in phase-separated droplets. If this is indeed the case, cells that carry HRD mutations might be less able than wildtype cells to mount effective transcriptional responses to DNA damage or viral infection, or might fail to maintain cell-type-specific geneexpression programs over time. Such defects could contribute to cancer and other diseases. To our knowledge, any clinical manifestations of HRD mutations are unknown, and await discovery.

$\mathrm{P}-\mathrm{TEFb}$ phosphorylates other transcriptional regulatory proteins in addition to Pol II (ref. 9). As such, inhibition of P-TEFb kinase activity shows promise as a therapeutic strategy for combating a variety of diseases, including HIV infection and cancer ${ }^{10}$. Compounds that inhibit $\mathrm{P}-\mathrm{TEFb}$ repress transcription across the entire genome ${ }^{3}$, and it has been assumed that the inhibitors alter the function of P-TEFb's numerous targets for phosphorylation ${ }^{9}$. Is phase separation also involved? Phase-separated domains can reversibly form and dissolve, and this behaviour is sensitive to phosphorylation ${ }^{11}$. Notably, Pol II CTD phosphorylation seems to either promote or prevent phase separation, depending on which other proteins are present in the droplet ${ }^{1,4,5}$. Inhibition of $\mathrm{P}-\mathrm{TEFb}$ kinase activity might therefore alter the formation and dissolution of phase-separated droplets. This intriguing possibility remains to be rigorously tested.

James A. Goodrich and Dylan J. Taatjes are in the Department of Chemistry and Biochemistry, University of Colorado, Boulder, Colorado 80303, USA.

e-mails: james.goodrich@colorado.edu; dylan.taatjes@colorado.edu

1. Lu, H. et al. Nature 558, 318-323 (2018).

2. Ebmeier, C. C. et al. Cell Rep. 20, 1173-1186 (2017)

3. Zhou, Q., Li, T. \& Price, D. H. Annu. Rev. Biochem. 81, 119-143 (2012).

4. Kwon, l. et al. Cell 155, 1049-1060 (2013).

5. Boehning, M. et al. Preprint at bioRxiv http://dx.doi. org/10.1101/316372 (2018).

6. Larson, A. G. et al. Nature 547, 236-240 (2017).

7. Strom, A. R. et al. Nature 547, 241-245 (2017).

8. Hnisz, D., Shrinivas, K., Young, R. A.,

Chakraborty, A. K. \& Sharp, P. A. Cell 169, 13-23 (2017).

9. Sanso, M. et al. Genes Dev. 30, 117-131 (2016).

10.Ferguson, F. M. \& Gray, N. S. Nature Rev. Drug Disc. 17, 353-377 (2018).

11.Li, P. et al. Nature 483, 336-340 (2012).

This article was published online on 30 May 2018. 